\title{
Lesiones transmediastinales. Revisión de la literatura y una visión de lo que se debe de hacer
}

\author{
Alberto Pérez Cantú-Sacal, $₫$ Demian Trueba-Lozano, David Roland de Rungs-Brown, \\ Juan Carlos Vázquez-Minero
}

Hospital Central de la Cruz Roja Mexicana I.A.P., Ciudad de México.

Trabajo recibido: 15-V-2015; aceptado: 28-V-2015

\begin{abstract}
RESUMEN. Introducción: El trauma de tórax transmediastinal es una causa importante de ingreso hospitalario; ha ocurrido un aumento de los mismos con el transcurso del tiempo gracias a la mejora en atención prehospitalaria. El concepto de «la hora dorada» y los nuevos protocolos de transporte «scoop and run» se han visto reflejados en un mayor número de pacientes que arriban a los servicios de urgencias con vida (de 44-53\% con estabilidad hemodinámica). Objetivo: Exponer la problemática a la que se enfrenta el personal de cirugía de urgencias ante los pacientes con lesiones transmediastinales, revisar la literatura mundial para protocolizar la atención primaria de manera que se puedan demostrar los pasos correctos de diagnóstico y tratamiento para evitar mayor morbilidad y mortalidad. Material y métodos: Se llevó a cabo una búsqueda de información en PubMed de literatura restringida a artículos originales en español, inglés y francés con las palabras MESH en forma entrecruzada «transmediastinal trauma», «transmediastinal injury», «transmediastinal gunshot» sobre el manejo de estas lesiones. Se obtuvo un total de 33 artículos y se utilizaron las series de casos más representativas de los últimos 35 años para revisar de manera sistematizada la literatura. Resultados: Para términos del presente estudio se tomaron en cuenta 15 artículos originales publicados entre 1981 y 2013, todos ellos con nivel de evidencia III (como lo dispone la United States Agency for Health Research and Quality). De estos artículos, 8 son estudios de pacientes, de los cuales 3 son prospectivos y nos permiten hacer un análisis en cuanto a mortalidad y éxito en las técnicas de diagnóstico y manejo empleados. Discusión y conclusiones: Después de analizar a 602 pacientes con lesiones transmediastinales, 46.8\% llegó al departamento de Urgencias con estabilidad hemodinámica, 53.1\% se presentó con inestabilidad y $25 \%$ de ellos fue sometido a toracotomía de reanimación y (como se ve reflejado en los porcentajes) muy cerca del total de los mismos fue trasladado a quirófano (51.4\%) con una mortalidad total de $41.6 \%$, lo cual implica que del total de los pacientes que presentan lesiones transmediastinales (que arriban al hospital) aproximadamente uno de cada dos fallece. Las lesiones transmediastinales pueden parecer abrumadoras en todos los casos; sin embargo, un abordaje sistematizado evitará, tanto cirugías innecesarias como la posibilidad de pasar por alto lesiones ocultas. Consideramos que todo médico de primer contacto que se enfrenta a lesiones transmediastinales, se deberá hacer las mismas preguntas que planteamos y tomar en cuenta las secuencias actuales de manejo, para que de esta manera sea capaz de atender a pacientes con trauma en mediastino que ultimadamente puedan ser dados de alta de manera rápida y satisfactoria.
\end{abstract}

Palabras clave: Lesiones transmediastinales, trauma torácico, trauma transmediastinal.

ABSTRACT. Introduction: Transmediastinal trauma has been an important cause of admission to emergency rooms, specially recently, due to improvements in pre-hospital care and the new «scoop and run» protocols have reflected in even more patients that arrive alive and with hemodynamic stability (44-53\%). Objective: We intend to review series of patients all over the world with transmediastinal trauma so we can demonstrate the situations that doctors and surgeons in the emergency rooms endure. We present a problem of this sort and questions that every physician should answer when diagnosing and treating transmediastinal injuries with a review of the most representative cases of this subject to gain a wide optic when resolving problems in trauma patients. Material and methods: We conducted a search in PubMed with MESH interlinked words «transmediastinal trauma», "transmediastinal injury», "transmediastinal gunshot» for a systematical review of the literature. We included articles in English, Spanish and French, only original texts from the last 35 years, together with highly cited publications that seemed necessary for full understanding. Moreover, we included several sets of guidelines to respond the questions we present. Results: A total of 15 original articles were taken into consideration, published between 1981 and 2013, all of them with level III of evidence according to the United States Agency for Health Research Quality; 8 articles were patient reviews, from which 3 were prospective studies thus giving us the chance to evaluate mortality and analyze diagnostic and therapeutic success in order to arrive to an informed conclusion about the best way to handle transmediastinal injuries. Discussion and conclusions: After a careful analysis of the aforementioned reviews, 602 patients with transmediastinal trauma were studied, $46.8 \%$ arrived to the emergency department with clinical hemodynamic stability, $53.1 \%$ presented with instability and $25 \%$ of them were submitted to an emergency department thoracotomy, 51.4 were transferred to an operating room for emergent surgery with an overall mortality of $41.6 \%$ which means that almost 1 out of every 2 patients with transmediastinal trauma eventually dies. Transmediastinal injuries may seem overwhelming every time, never the less, an organized and logical diagnostic approach will spare patients from unnecessary surgeries and untreated occult lesions. Furthermore we consider that every emergency room physician that encounters with transmediastinal trauma should ask himself the same questions we present in this review, this way we will be able to adequately treat and ultimately discharge patients free from complications.

Key words: Transmediastinal trauma, transmediastinal injury, transmediastinal gunshot. 


\section{INTRODUCCIÓN}

El trauma de tórax transmediastinal es una causa importante de ingreso hospitalario; ha ocurrido un aumento de los mismos con el transcurso del tiempo gracias a la mejora en atención prehospitalaria. El concepto de «la hora dorada» y los nuevos protocolos de transporte «scoop and run" se han visto reflejados en mayor número de pacientes que arriban a los servicios de urgencias con vida (de 44-53\% con estabilidad hemodinámica). ${ }^{1}$ Resulta interesante que -aún en esta época de avanzadas pruebas diagnósticasel criterio de abordaje diagnóstico y terapéutico adecuado de estas lesiones sigue siendo un desafío para el médico de primer contacto. Poca controversia gira alrededor del manejo de pacientes inestables, pues el diagnóstico no representa un reto y deben ser llevados a quirófano cuanto antes; sin embargo, todavía existe debate sobre la manera en la cual se diagnostica y maneja a pacientes con estabilidad hemodinámica (tabla 1). La siguiente viñeta clínica representa este problema y demuestra el proceso que debemos seguir ante dicho panorama clínico.

Paciente masculino de 35 años de edad, sin antecedentes de relevancia para el padecimiento actual quien ingresa

Tabla 1. Estado de inestabilidad hemodinámica.

1. Paro cardiorrespiratorio o criterios que demanden toracotomía de reanimación

2. Tamponade cardíaco

3. Estado persistente de choque hipovolémico ATLS* grado III a pesar de reanimación (pérdida hemática de 1,5002,000 , pulso > 120, presión arterial sistólica disminuida)

4. Hemotórax masivo

${ }^{*}$ ATLS: Advanced trauma life support. al servicio traído por paramédicos 30 minutos después de sufrir una herida por proyectil de arma de fuego perforante en extremidad superior izquierda y penetrante en hemitórax izquierdo, sin orificio de salida. Se valora en sala de choque: A-vía aérea permeable, B-ventilación espontánea, sin patrones patológicos, C-hemodinámicamente estable. En la valoración secundaria se observa herida por proyectil de arma de fuego a nivel de tercer espacio intercostal línea media axilar izquierda, sin orificio de salida, hipoventilación basal bilateral con predominio del lado izquierdo, ruidos cardíacos íntegros y sin agregados, abdomen asignológico, extremidad torácica izquierda con orificio de entrada y salida en sedal a nivel de tercio superior del brazo. Los estudios relevantes a su ingreso fueron: $\mathrm{PO}_{2} 96.1, \mathrm{PCO}_{2} 33.3, \mathrm{pH}$ 7.33, $\mathrm{BE}-7, \mathrm{HCO}_{3} 17.5, \mathrm{SO}_{2} 96.7 \%$. Los estudios de gabinete: FAST negativo, radiografía portátil de tórax AP con imagen compatible con hemotórax bilateral y tomografía computada donde se aprecia trayecto transmediastinal de proyectil y aire libre en mediastino (figura 1), esofagograma que demuestra fuga del medio de contraste en la porción torácica del esófago (figura 2).

Después del evento quirúrgico nos planteamos las siguientes interrogantes para motivos de este estudio.

1. En pacientes con evidencia de trauma transmediastinal con estabilidad hemodinámica, ¿cuál debería ser la conducta a seguir del médico de primer contacto en sala de choque?

2. ¿Cuáles son los estudios necesarios para descartar lesiones en estos pacientes y -de manera más importante- cuál es la secuencia correcta para llevarlos a cabo?

3. En caso de que el paciente no fuera candidato a tratamiento quirúrgico, ¿qué pasos debemos de

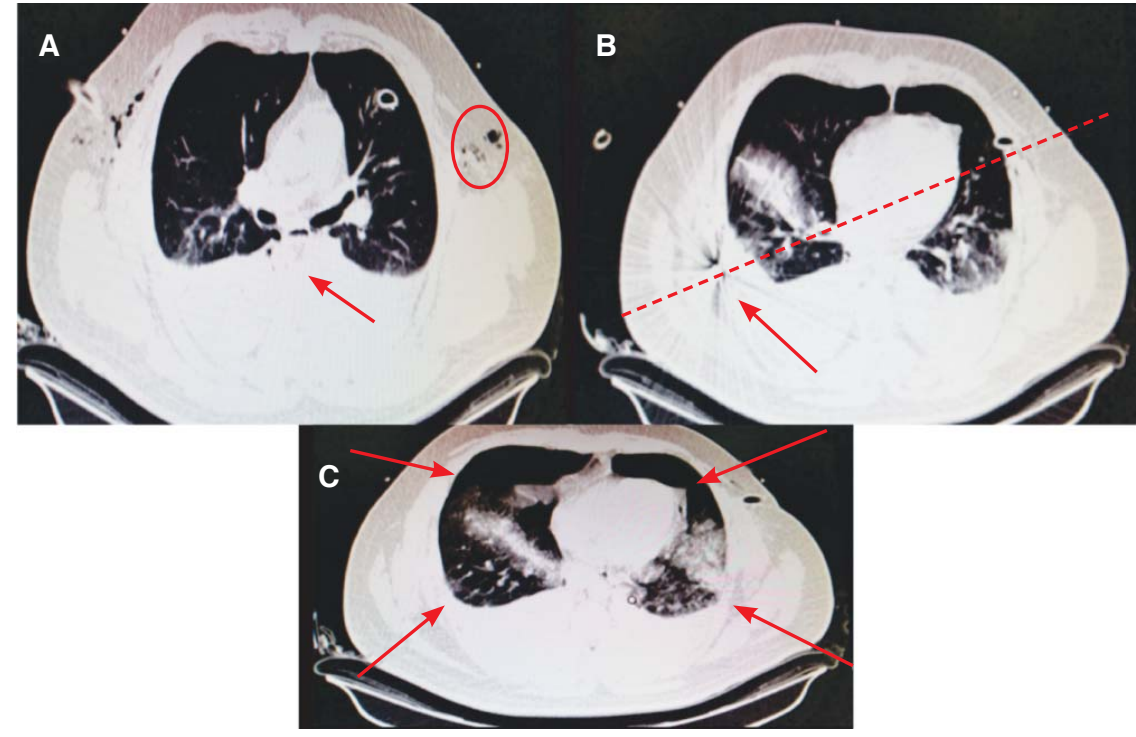

Figura 1.

Tomografía computada. A) círculo: enfisema subcutáneo. Flecha: neumomediastino; B) línea discontinua: trayecto probable del proyectil. Flecha: proyectil alojado en cavidad; C) flechas: delimitación de contusión pulmonar por la quemadura y trayecto del proyectil. 

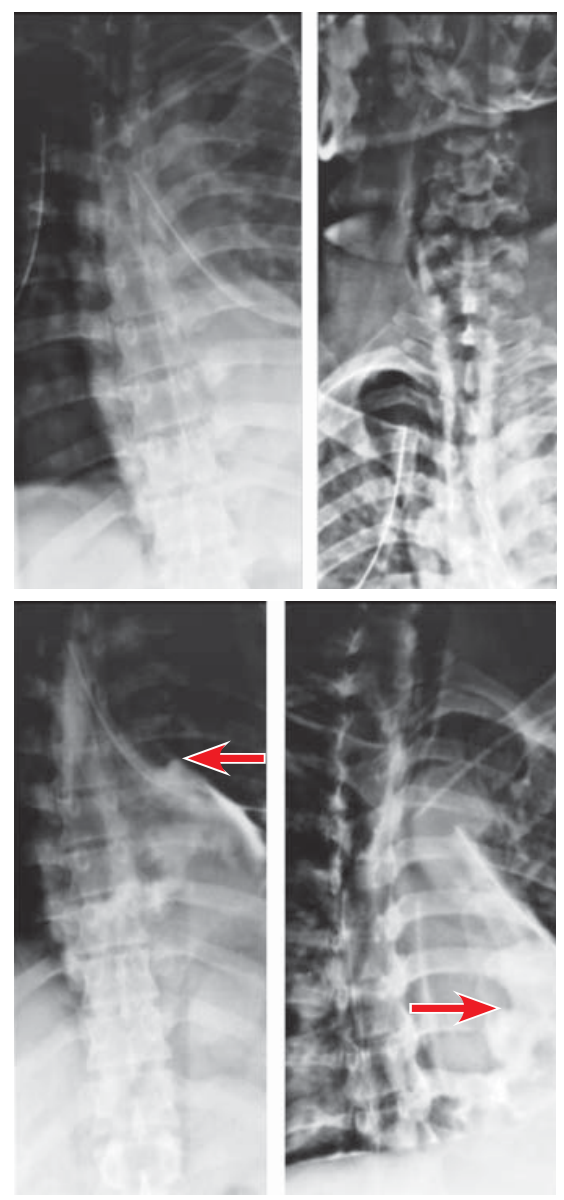

Figura 2.

Esofagograma donde se muestra claramente material de contraste extraesofágico (flecha).

seguir para su vigilancia y cuáles son los parámetros a tomar en cuenta para evitar complicaciones?

4. Cuando el paciente es candidato a tratamiento quirúrgico, ¿qué abordaje debemos de efectuar, tomando en cuenta las lesiones encontradas y qué se debe de hacer?

En nuestro caso se optó por manejo quirúrgico de la lesión debido a los resultados del proceso diagnóstico: diagnóstico prequirúrgico: herida por proyectil de arma de fuego penetrante en tórax, hemotórax bilateral y lesión esofágica en tercio inferior. Cirugía proyectada: toracotomía anterolateral izquierda. Diagnóstico posquirúrgico: lesión grado II en diafragma, lesión grado I de estómago en la curvatura mayor, lesión esofágica en tercio distal lateral izquierdo de $2 \mathrm{~cm}$ con compromiso del $30 \%$ de la circunferencia. Cirugía efectuada: gastrorrafia, frenorrafia, esofagorrafia primaria longitudinal con parche de pleura parietal mediastínica pediculado. Con esto se logró adecuado control de las lesiones y no existieron complicaciones derivadas del proceso diagnóstico y terapéutico.

\section{MATERIAL Y MÉTODOS}

Se llevó a cabo una búsqueda de información en PubMed de literatura restringida a artículos originales en español,

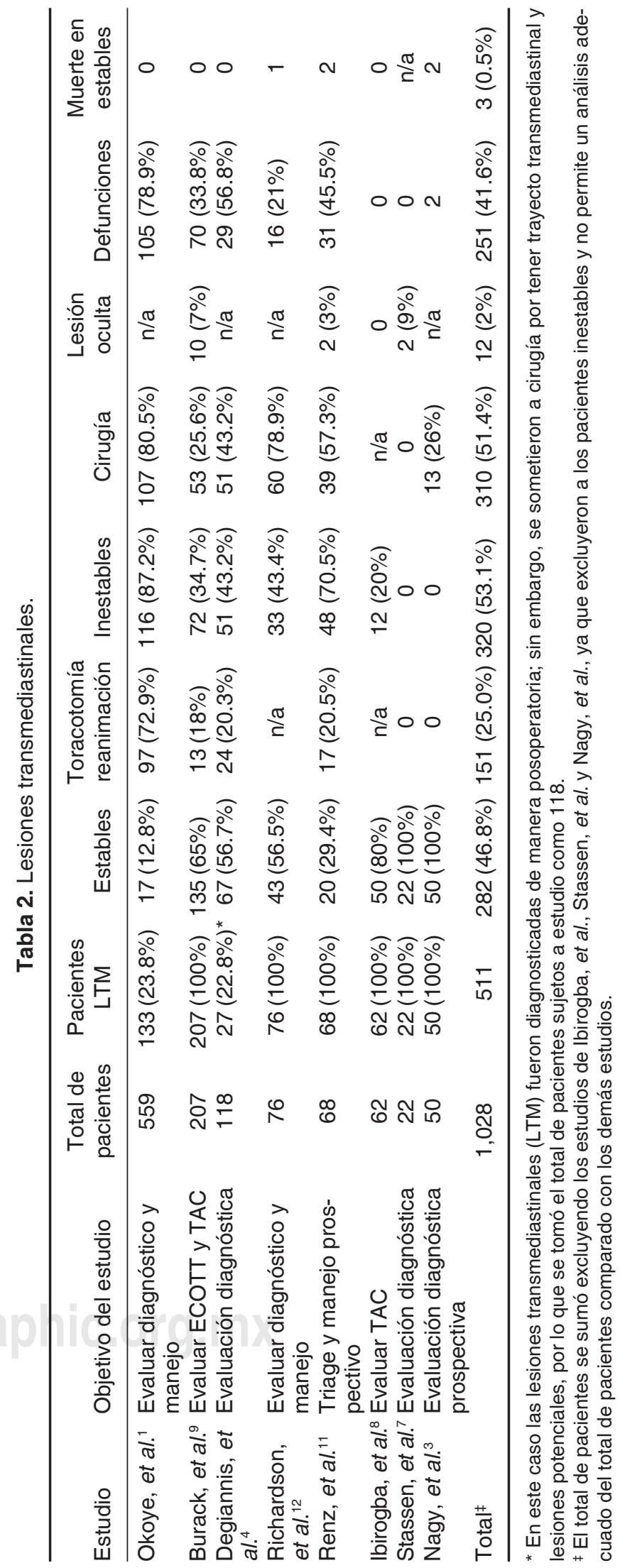


inglés y francés con las palabras MESH en forma entrecruzada «transmediastinal trauma», "transmediastinal injury», "transmediastinal gunshot» sobre el manejo de estas lesiones. Se obtuvo un total de 33 artículos y se utilizaron las series de casos más representativas de los últimos 35 años para revisar de manera sistematizada la literatura. Se construyó una tabla de las series retrospectivas y prospectivas con más influencia y volumen para efectuar un análisis detallado y metodológico de las conductas diagnósticas y terapéuticas empleadas hoy en día.

\section{RESULTADOS}

Para términos del presente estudio se tomaron en cuenta 15 artículos originales publicados entre 1981 y 2013, todos ellos con nivel de evidencia III (como lo dispone la United States Agency for Health Research and Quality). De estos artículos, 8 eran estudios de pacientes, de los cuales 3 son prospectivos y nos permiten hacer un análisis en cuanto a mortalidad y éxito en las técnicas de diagnóstico y manejo empleados (tabla 2); posteriormente, se completó la revisión de artículos actuales que hablan de lesiones transmediastinales, mismos que permitieron contestar las preguntas planteadas de la mejor manera posible. Lo obtenido es presentado a continuación:

Pregunta 1: En pacientes con evidencia de trauma transmediastinal con estabilidad hemodinámica, ¿cuál debería ser la conducta a seguir del médico de primer contacto en sala de choque?
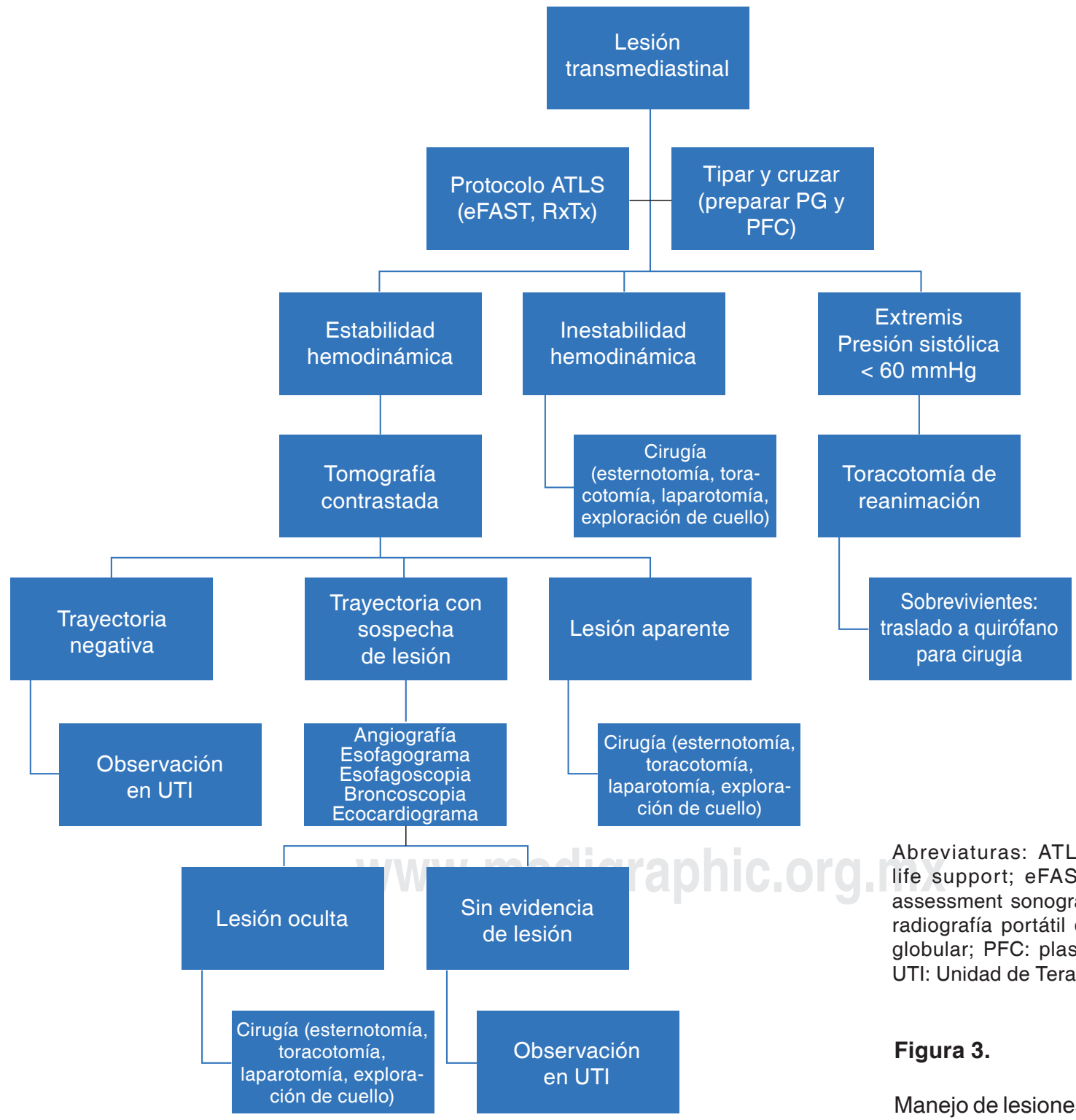

Abreviaturas: ATLS: advanced trauma life support; eFAST: extended focused assessment sonography for trauma; RxTx: radiografía portátil de tórax; PG: paquete globular; PFC: plasma fresco congelado; UTI: Unidad de Terapia Intensiva.

\section{Figura 3.}

Manejo de lesiones transmediastinales. 
Los pacientes con lesiones penetrantes transmediastinales hemodinámicamente estables forman un grupo muy selecto con un potencial muy elevado de lesiones ocultas. Por razones que pueden ser obviadas se deberán de tomar en cuenta los protocolos de ATLS para toda situación de trauma.

De manera inicial, como lo estipula un amplio estudio de balística y trauma en población civil realizado por Lichte et al., es necesario descartar primero la existencia de neumotórax o hemotórax mediante una radiografía de tórax y/o un eFAST, para que de esta forma sea posible llevar a cabo evaluaciones más específicas de acuerdo con las condiciones del paciente y como se presente la evolución del mismo (figura 1). ${ }^{1,2}$

De acuerdo con varias publicaciones y después de un cuidadoso consenso, los pacientes deberán de ser catalogados en tres grupos de estudio: el primero, pacientes que cuentan con presión sistólica de más de $100 \mathrm{mmHg}$ en los cuales (de manera primaria) la evaluación deberá de incluir radiografía de tórax, ecocardiograma y tomografía computada con contraste intravenoso; la mayoría de las veces, el manejo de estos pacientes será observación y en pocos casos, cirugía. El segundo grupo incluye aquellos pacientes con presión sistólica de $60-100 \mathrm{mmHg}$, en los cuales la evaluación inicial debe de efectuarse mediante exploración física, radiografía de tórax y de ser posible ecocardiograma; el manejo más frecuente será operatorio por medio de una toracotomía anterolateral izquierda. El tercer grupo de pacientes abarca aquellos con una presión sistólica menor de $60 \mathrm{mmHg}$, en quienes de primera instancia está indicada una toracotomía de reanimación. ${ }^{3}$

Pregunta 2: ¿Cuáles son los estudios necesarios para descartar lesiones en estos pacientes $y$-de manera más importante- cuál es la secuencia correcta para llevarlos a cabo?

En el escrito realizado por Nagy et al., se reporta que $16 \%$ de las lesiones ocultas significativas se encontraron en sujetos hemodinámicamente estables, mientras que en los estudios de Degiannis et al. y Richardson et al., se encontró un 42 y $63 \%$ de estos pacientes, respectivamente. ${ }^{3,4}$

Por otra parte, en el estudio realizado por Okoye et al. se estipula que tradicionalmente los estudios que deben efectuarse a pacientes estables deberían incluir inicialmente aortografía, esofagoscopia, broncoscopia y ecocardiograma de manera rutinaria, con el fin de descartar lesiones aerodigestivas y vasculares. Sin embargo, en la última década se ha utilizado de manera preferencial la tomografía computada con contraste intravenoso (en muchos casos [67\%] como único estudio) para determinar la trayectoria del proyectil y por consiguiente, los órganos lesionados. Una vez obtenido el estudio y determinada una trayectoria sospechosa, también se puede efectuar en pacientes estables una toracoscopia, la cual ha ganado más aceptación debido a su alta sensibilidad para detectar lesiones inadvertidas cuando una tomografía demuestra trayectos con sospechas. ${ }^{1-3}$

Continúa existiendo cierta incertidumbre en cuanto a los pasos a seguir en el proceso diagnóstico; aunque actualmente queda muy claro que el primer paso en un paciente estable es la obtención de una tomografía, existe poca evidencia sobre que resulta más adecuado después. Todo parece indicar que cuando encontramos un trayecto que parece comprometer el mediastino posterior - donde se encuentran los grandes vasos y las estructuras aerodigestivas - primero se lleven a cabo angiografía, esofagograma, esofagoscopia y broncoscopia; cuando la lesión compromete el mediastino anterior, primero se debe efectuar un ecocardiograma transtorácico. ${ }^{3}$

Finalmente es necesario tomar en cuenta el estudio realizado por Stassen et al., donde se observa claramente que muchas veces una tomografía realizada de forma adecuada es de utilidad para frenar en este punto la batería de estudios, disminuyendo la morbilidad, pues los estudios de seguimiento son invasivos y sus costos son altos. En este grupo de pacientes, los que fueron seleccionados para ser valorados de esta manera reflejaron una disminución de costos desde hasta US $\$ 5,360$ con una media de disminución de US $\$ 2,173$, es decir, debemos ser cuidadosos y no caer en la creencia antigua de que todos los pacientes deben de ser sometidos a los mismos estudios. ${ }^{4}$ En la actualidad, este concepto ha sido reemplazado al demostrar el éxito en valoraciones como la de Stassen; p. ej.; un ultrasonido realizado por alguien con la habilidad suficiente, cuenta con sensibilidad por arriba de 95\% para diagnosticar hemopericardio, y la necesidad de llevar a cabo una ventana pericárdica se torna obsoleta.5,6

Pregunta 3: En caso de que el paciente no fuera candidato a tratamiento quirúrgico, ¿qué pasos debemos de seguir para su vigilancia y cuáles son los parámetros a tomar en cuenta para evitar complicaciones?

Los pacientes estables que de entrada no presenten algún dato que demande corrección quirúrgica deberán ser sometidos a una tomografía, como se mencionó con anterioridad. De acuerdo con lo publicado en el reporte elaborado por Ibirogba et al. una tomografía se determina concluyentemente negativa cuando no existe un hematoma mediastinal, no se encuentra aire libre en el mediastino y la trayectoria del proyectil está claramente lejos de cualquier estructura mediastinal. Por otra parte, se considera un estudio no concluyente cuando hay un hematoma mediastinal contenido, pneumomediastino o proximidad del 
trayecto del proyectil a cualquier estructura mediastinal; es específicamente en este grupo de pacientes en los cuales debe procederse con cautela y completar su valoración con una angiografía, esofagograma (o esofagoscopia cuando esté disponible) y ecocardiograma, como mínimo (en este caso se dejó la broncoscopia solamente para evaluar pacientes con alguna sintomatología respiratoria, aunque si se encuentra un trayecto sospechoso sugerimos realizarlo rutinariamente) para que en caso de resultar normales, se cumpla con vigilancia en una Unidad de Cuidados Intensivos por un período no menor de 24 a 48 horas. ${ }^{7,8}$

Pregunta 4: Cuando el paciente es candidato a tratamiento quirúrgico, ¿qué abordaje debemos efectuar tomando en cuenta las lesiones encontradas y qué se debe de hacer?

El abordaje quirúrgico en estas situaciones dependerá siempre del estado hemodinámico en el cual se encuentre el paciente. En aquellos sujetos que se encuentren in extremis (presión sistólica $<60 \mathrm{mmHg}$ ) siempre será necesaria una toracotomía de reanimación en sala de choque con pinzamiento de la aorta para control del sangrado.

En pacientes con lesiones cardíacas o de grandes vasos, la incisión preferida para obtener mejor exposición es la esternotomía media pues permite visualizar de manera adecuada el mediastino anterior y medio (en situaciones de emergencia se cuestiona por disponibilidad limitada del material para su adecuada y pronta realización). Ahora bien, cuando existen lesiones en la porción posterior del mediastino (esofágicas, traqueobronquiales o en la aorta descendente), el abordaje preferido será la toracotomía: toracotomía izquierda, para un acceso óptimo hacia aorta, vasos subclavios izquierdos y esófago distal, toracotomía derecha para acceso óptimo hacia tráquea, carina y esófago medio.

La incisión más versátil en situaciones de trauma se lleva cabo con el paciente en posición supina mediante una incisión anterolateral en cuarto o quinto espacio intercostal de un lado del tórax para que exista potencial para extender la incisión hacia el lado contralateral (toracotomía tipo «clam-shell»), permitiendo así exponer los grandes vasos y el contenido en ambos lados del tórax. En los pacientes que tengan sondas endopleurales como consecuencia de la lesión, el lado de la toracotomía será aquel del cual se obtenga mayor gasto a través de la sonda.

\section{DISCUSIÓN}

Históricamente las heridas transmediastinales precipitan inestabilidad hemodinámica $(43 \%$ de los pacientes que arriban al área de urgencias) ya que se asocian con lesión vascular en $50 \%$ de los casos, requieren cirugía urgente en $35-60 \%$ de las ocasiones y se asocian con una mortalidad transoperatoria del $20-40 \%{ }^{3,9,10}$

Considerando las estadísticas antes mencionadas, hay que tomar en cuenta lo estipulado en los estudios prospectivos realizados por Richardson et al. y Burack et al., en los cuales los pacientes con heridas transmediastinales que ingresan con presión sistólica mayor de $100 \mathrm{mmHg}$ sin sangrado evidente, pueden ser sometidos a la batería de pruebas diagnósticas con seguridad, pues el desarrollo de inestabilidad en un paciente que arriba estable ocurre en menos del $3 \%$ de las ocasiones. En consecuencia, vale la pena mencionar que los pacientes que llegan con una presión sistólica de 60-100 mmHg y se recuperan después de reanimación con líquidos, sin evidencia de sangrado no ameritan cirugía urgente y pueden ser evaluados sin complicaciones en presencia de monitoreo continuo, con base en lo reportado por Renz et al.11

Es importante considerar las series de casos más representativas que se han publicado en la literatura mundial cuando se evalúa este tipo de pacientes. Al presente, han cambiado los pasos diagnósticos y es importante puntualizar como lo reportaron Nagy et al., (quienes realizaron una comparación de los signos vitales en pacientes con lesiones mediastinales y los que no la tuvieron) que puede no existir diferencia en presión sistólica, pulso, $\mathrm{pH}$, déficit de base o gasto de las sondas endopleurales, lo cual nos traduce que hasta no tener evidencia del trayecto del proyectil y estudios que descarten la posibilidad de alguna lesión, no podemos detener el proceso diagnóstico y debemos de tratar a los pacientes como si algún daño estuviera presente. Es aquí de suma importancia donde se vuelve obligatorio el diagnóstico de lesiones aerodigestivas, el cual siempre toma tiempo (idealmente debería de ocurrir en un período menor de 8 horas, siempre después de descartar cualquier lesión vascular) ${ }^{4}$ y la cabida de nuevos procesos diagnósticos como el empleo de toracoscopia para evaluación de lesiones, inclusive el uso de la misma para realizar ventanas pericárdicas con mínima invasión.,10-15

Después de una evaluación de los estudios mencionados para propósitos de este artículo (tabla 2), podemos afirmar que después de analizar a 602 pacientes con lesiones transmediastinales, $46.8 \%$ llegó al departamento de Urgencias con estabilidad hemodinámica, $53.1 \%$ se presentó con inestabilidad y $25 \%$ de ellos fue sometido a toracotomía de reanimación y (como se ve reflejado en los porcentajes) muy cerca del total de los mismos fue trasladado a quirófano (51.4\%) con una mortalidad total de $41.6 \%$, lo cual implica que del total de los pacientes que presentan lesiones transmediastinales (que arriban al hospital) aproximadamente uno de cada dos fallece. Las lesiones ocultas y muerte en pacientes estables son menores en cuanto a porcentajes ( 2 y $0.5 \%$, respectivamente) gracias a los pasos diagnósticos que se han propuesto en los mejores centros 
de trauma del mundo que se presentan en este estudio. Extrapolando los resultados de este análisis, se puede efectuar una comparación con los porcentajes mencionados sin encontrar variaciones importantes con la literatura mundial, que se explica por la cantidad de pacientes que muere a pesar de cualquier conducta médica como consecuencia de la gravedad de sus lesiones. ${ }^{10}$

\section{CONCLUSIONES}

La causa más frecuente de muerte relacionada con trauma en la primera hora de hospitalización globalmente incluye a las heridas que causan daño neurológico o a la vasculatura torácica. De manera general, los pacientes con esta clase de heridas se dividen de forma bastante lógica y no se requiere mucha perspicacia al principio, para discernir entre los que requieren intervención quirúrgica y los que pueden ser evaluados y observados.

De igual manera serán necesarias evaluaciones prospectivas en un futuro, así como de seguimiento en la cirugía de pacientes estables y sus complicaciones. Contamos con mucha información acerca del protocolo diagnóstico y de conducta en las primeras horas de estancia hospitalaria; sin embargo, falta información y reporte a largo plazo de estos pacientes y no se tiene una estimación de los mismos por limitaciones de los estudios encontrados. Tenemos lagunas en la información de lesiones inadvertidas en pacientes inestables y las defunciones que éstas causan; es decir, queda en duda la mejor manera de evitarlas cuando nos encontramos en quirófano.

Las lesiones transmediastinales pueden parecer abrumadoras en todos los casos; aunque, un abordaje sistematizado evitará tanto cirugías innecesarias como la posibilidad de pasar por alto lesiones ocultas. La mecánica correcta de valoración de los pacientes que tengan alta sospecha de lesiones ocultas en el mediastino y el abordaje de las mismas de manera expedita (como lo expusimos en este escrito) bien podría cambiar las tasas de mortalidad que observamos hoy en día en pacientes estables, disminuyendo el número de complicaciones.

Consideramos que todo médico de primer contacto que enfrenta lesiones transmediastinales deberá hacer las mismas preguntas que nosotros planteamos, y tomar en cuenta las secuencias actuales de manejo para ser capaz de atender pacientes con trauma en mediastino que ultimadamente puedan ser dados de alta de manera rápida y satisfactoria.

\section{REFERENCIAS}

1. Okoye OT, Talving P, Texeira PG, et al. Transmediastinal gunshot wounds in a mature trauma centre: chang- ing perspectives. Injury 2013;44(9):1198-1203. doi: 10.1016/j.injury.2012.12.014.

2. Lichte P, Oberbeck R, Binnebösel M, Wildenauer R, Pape HC, Kobbe P. A civilian perspective on ballistic trauma and gunshot injuries. Scand J Trauma Resusc Emerg Med 2010;18:35. doi: 10.1186/1757-7241-18-35.

3. Nagy KK, Roberts RR, Smith RF, et al. Trans-mediastinal gunshot wounds: are "stable" patients really stable? World J Surg 2002;26(10):1247-1250.

4. Degiannis E, Benn CA, Leandros E, Goosen J, Boffard K, Saadia R. Transmediastinal gunshot injuries. Surgery 2000;128(1):54-58.

5. Zarain-Obrador L, Al-Lal YM, de Tomás-Palacios J, Amunategui-Prats I, Turégano-Fuentes F. Transmediastinal and transcardiac gunshot wound with hemodynamic stability. Case Rep Surg 2014;2014:985097. doi: 10.1155/2014/985097.

6. Rozycki GS, Feliciano DV, Ochsner MG, et al. The role of ultrasound in patients with possible penetrating cardiac wounds: a prospective multicenter study. J Trauma 1999;46(4):543-551.

7. Stassen NA, Lukan JK, Spain DA, et al. Reevaluation of diagnostic procedures for transmediastinal gunshot wounds. J Trauma 2002;53(4):635-638.

8. Ibirogba S, Nicol AJ, Navsaria PH. Screening helical computed tomographic scanning in haemodynamic stable patients with transmediastinal gunshot wounds. Injury 2007;38(1):48-52.

9. Burack JH, Kandil E, Sawas A, et al. Triage and outcome of patients with mediastinal penetrating trauma. Ann Thorac Surg 2007;83(2):377-382.

10. Bastos R, Baisden CE, Harker L, Calhoon JH. Penetrating thoracic trauma. Semin Thorac Cardiovasc Surg 2008;20(1):19-25. doi: 10.1053/j.semtcvs.2008.01.003.

11. Renz BM, Cava RA, Feliciano DV, Rozycki GS. Transmediastinal gunshot wounds: a prospective study. J Trauma 1999;48(3):416-421.

12. Richardson JD, Flint LM, Snow NJ, Gray LA Jr, Trinkle JK. Management of transmediastinal gunshot wounds. Surgery 1981;90(4):671-676.

13. Asensio JA, Berne J, Demetriades D, et al. Penetrating esophageal injuries: time interval of safety for preoperative evaluation-how long is safe? J Trauma 1997;43(2):319-324.

14. Asensio JA, Chahwan S, Forno W, et al.; American Association for the Surgery of Trauma. Penetrating esophageal injuries: multicenter study of the American Association for the Surgery of Trauma. J Trauma 2001;50(2):289-296.

15. Casós SR, Richardson JD. Role of thoracoscopy in acute management of chest injury. Curr Opin Crit Care 2006;12(6):584-589.

\section{$\bowtie$ Correspondencia:}

Dr. Alberto Pérez Cantú-Sacal

Hospital Central de la Cruz Roja Mexicana I.A.P. Av. Ejército Nacional Núm. 1032, Col. Los Morales Polanco, 11510, Del. Miguel Hidalgo, México, D.F. Tel. 53951111, ext. 214

Correo electrónico: dr.apcantu@gmail.com

Los autores declaran no tener conflicto de intereses. 